\title{
Generalized Macdonald polynomials, spectral duality for conformal blocks and AGT correspondence in five dimensions
}

\author{
Yegor Zenkevich \\ ITEP, \\ 25 Bolshaya Cheremushkinskaya street, Moscow, Russia \\ Institute for Nuclear Research of the Russian Academy of Sciences, \\ 7 a Prospekt 60-letiya Oktyabrya, Moscow, Russia \\ NRNU Moscow Engineering Physics Institute, \\ 31 Kasirskoe chaussee, Moscow, Russia \\ E-mail: yegor.zenkevich@gmail.com
}

ABSTRACT: We study five dimensional AGT correspondence by means of the $q$-deformed beta-ensemble technique. We provide a special basis of states in the $q$-deformed CFT Hilbert space consisting of generalized Macdonald polynomials, derive the loop equations for the beta-ensemble and obtain the factorization formulas for the corresponding matrix elements. We prove the spectral duality for SU(2) Nekrasov functions and discuss its meaning for conformal blocks. We also clarify the relation between topological strings and $q$-Liouville vertex operators.

KEYwords: Matrix Models, Duality in Gauge Field Theories, Conformal and W Symmetry

ARXIV EPRINT: 1412.8592 


\section{Contents}

1 Introduction 1

2 Generalized Macdonald polynomials 5

3 q-deformed Dotsenko-Fateev integrals $\quad 8$

4 Loop equations for $q$-deformed beta-ensemble 9

5 Spectral duality for conformal blocks $\quad 12$

6 Comparison with topological strings 13

$\begin{array}{lll}7 & \text { Conclusions } & 14\end{array}$

A Macdonald polynomials and Ruijsenaars Hamiltonians 15

$\begin{array}{ll}\text { B Five dimensional Nekrasov functions and AGT relations } & 17\end{array}$

$\begin{array}{ll}\text { C Ruijsenaars Hamiltonians and loop equations } & 18\end{array}$

$\begin{array}{ll}\text { D Useful identities } & 19\end{array}$

\section{Introduction}

Original Alday-Gaiotto-Tachikawa conjecture [1-4] states that instanton partition functions of four dimensional $\mathcal{N}=2$ supersymmetric gauge theories in $\Omega$-background are equal to conformal blocks of the Liouville theory. This connection has provided insight in two dimensional CFT and gauge theory as well as other related areas [5-12]. In this paper we will be concerned with a generalization of this duality in which five dimensional $\mathcal{N}=1$ gauge theories correspond to the $q$-deformation of the Liouville or Toda field theories. This version of the AGT duality has been checked for many cases in [13]-[15]. We will focus on the simplest possible case of ( $q$-deformed) Virasoro four-point conformal block.

We briefly introduce the approach that we will use to study the five dimensional AGT correspondence. As in the four dimensional case the instanton part of the Nekrasov partition function for the $\mathrm{SU}(2)$ gauge theory with four fundamental hypermultiplets is given by a series in the (exponentiated) coupling constant $\Lambda$. Each term in the expansion has in fact a finer structure consisting of several factorized terms, so that the whole sum can be written as a sum over pairs of partitions $(A, B)$ :

$$
Z_{\mathrm{Nek}}\left(\Lambda \mid a, m_{f}, q, t\right)=\sum_{A, B} \Lambda^{|A|+|B|} \frac{\left(z_{\text {fund }}(A)\right)^{2}\left(z_{\text {fund }}(B)\right)^{2}}{z_{\text {vect }}(A, B)}
$$

where $z_{\text {fund,vect }}$ are certain polynomials in the gauge theory parameters which we write explicitly in appendix B. On the CFT side of the AGT correspondence this sum corresponds 
to the expansion of four-point conformal block in terms of a certain complete system of basis vectors $|A, B, \alpha\rangle$, labelled by pairs of partitions, which can be written schematically as

$$
\begin{aligned}
& \mathcal{B}\left(\Lambda \mid \begin{array}{ccc}
\alpha_{\Lambda} & \alpha_{1} \\
\alpha_{0} & \alpha & \alpha_{\infty}
\end{array}\right)=\left\langle V_{0}(0) V_{\Lambda}(\Lambda)\right| \text { through } V_{\alpha} \text { primary }\left|V_{1}(1) V_{\infty}(\infty)\right\rangle= \\
& =\sum_{A, B} \Lambda^{|A|+|B|}\left\langle V_{0}(0) V_{\Lambda}(1) \mid A, B, \alpha\right\rangle\left\langle A, B, \alpha \mid V_{1}(1) V_{\infty}(\infty)\right\rangle \stackrel{\mathrm{AGT}}{=} \\
& \stackrel{\mathrm{AGT}}{=} \sum_{A, B} \Lambda^{|A|+|B|} \frac{\left(z_{\text {fund }}(A)\right)^{2}\left(z_{\text {fund }}(B)\right)^{2}}{z_{\text {vect }}(A, B)} .
\end{aligned}
$$

One way to prove the AGT correspondence is to find the special basis for which the equality in the last two lines holds not only for each power of $\Lambda$, but for each pair of partitions [1618], so that

$$
\left\langle V_{0}(0) V_{\Lambda}(1) \mid A, B, \alpha\right\rangle\left\langle A, B, \alpha \mid V_{1}(1) V_{\infty}(\infty)\right\rangle \stackrel{\operatorname{AGT}}{=} \frac{\left(z_{\text {fund }}(A)\right)^{2}\left(z_{\text {fund }}(B)\right)^{2}}{z_{\text {vect }}(A, B)} .
$$

When $t=q$ (or $c=1$ in CFT) there is indeed a simple basis, consisting of Schur polynomials giving the desired expansion [19, 20]. However, for general $t, q$ the basis turns out to be a lot more elaborate: in particular the naive deformation of Schur polynomials to Macdonald polynomials is not enough. In this paper we show that the right basis is in fact given by the generalization of the Macdonald polynomials $M_{A B}$ depending on two partitions and an additional moduli parameter [23]. Similar polynomials for the four dimensional case (i.e. generalized Jack polynomials) were introduced in [21] and also studied in [22].

To compute the matrix elements in eq. (1.3) we use the $q$-deformed version of the Dotsenko-Fateev (DF) representation for the conformal block. After the $q$-deformation the cuts in the DF integrands pulverize into a set of poles, so that the integrals can be taken by residues. The sum over residues is captured by the Jackson $q$-integral, which is in fact a sum of the form

$$
\int_{0}^{a} d_{q} z f(z)=(1-q) \sum_{k \geq 0} q^{k} a f\left(q^{k} a\right)=\frac{1-q}{1-q^{a \partial_{a}}}(a f(a)) .
$$

The matrix elements in this framework are given by the $q$-deformed Selberg averages of the generalized Macdonald polynomials:

$$
\left\langle V_{0}(0) V_{\Lambda}(1) \mid A, B, \alpha\right\rangle=\frac{\int_{0}^{1} d_{q}^{N} x \mu(x) M_{A B}(x)}{\int_{0}^{1} d_{q}^{N} x \mu(x)},
$$

where $\mu(x)$ is certain $q$-deformed Selberg measure. To compute the averages we devise a set of loop equation for the $q$-deformed beta-ensemble (also called the $(q, t)$-matrix model) which provide the recurrence relations for the averages of any symmetric polynomials.

One of our main results is the remarkable factorized formula for the averages of generalized Macdonald polynomials (4.12). It can be written schematically as

$$
\frac{\int_{0}^{1} d_{q}^{N} x \mu(x) M_{A B}(x)}{\int_{0}^{1} d_{q}^{N} x \mu(x)}=\frac{\left(z_{\text {fund }}(A)\right)\left(z_{\text {fund }}(B)\right)}{\left[z_{\text {vect }}(A, B)\right]^{1 / 2}}
$$

and evidently leads to the AGT conjecture for four-point blocks (1.3). Though we were not able to obtain a rigorous proof of this formula, we have checked it for several lower 
polynomials. This is the only missing step in the proof of the five dimensional AGT conjecture, however the conceptual picture is already apparent.

Let us clarify the relation between our study and the alternative approach to the AGT duality proposed in $[24,25]$. In these works the sum over residues in the DF integrals without any basis decomposition was shown to be the sum over pairs of partitions:

$$
\begin{aligned}
\mathcal{B}( & \left(\Lambda \mid \begin{array}{cc}
\alpha_{\Lambda} & \begin{array}{c}
\alpha_{1} \\
\alpha_{0}
\end{array} \\
\alpha_{\infty}
\end{array}\right)=\left\langle V_{0}(0) V_{\Lambda}(\Lambda)\right| \text { through } V_{\alpha} \text { primary }\left|V_{1}(1) V_{\infty}(\infty)\right\rangle \simeq \\
& \simeq \int_{0}^{1} d_{q}^{N_{+}} x d_{q}^{N_{-}} y \mu(x) \mu(y) \nu(\Lambda, x, y)=\sum_{R_{+}, R_{-}} \mu\left(q^{R_{+}} t^{\rho}\right) \mu\left(q^{R_{-}} t^{\rho}\right) \nu\left(\Lambda, q^{R_{+}} t^{\rho}, q^{R_{-}} t^{\rho}\right),
\end{aligned}
$$

where $\nu(\Lambda, x, y)=\sum_{A B} \Lambda^{|A|+|B|} M_{A B}^{*}(x) M_{A B}(y)$ is a certain rational function and $\rho=$ $(N-1, N-2, \ldots, 0)$ is the Weyl vector. Moreover, the integrands turn out to combine miraculously into the Nekrasov partition function

$$
\mu\left(q^{R_{+}} t^{\rho}\right) \mu\left(q^{R_{-}} t^{\rho}\right) \nu\left(\Lambda, q^{R_{+}} t^{\rho}, q^{R_{-}} t^{\rho}\right)=\left(\Lambda^{\vee}\right)^{\left|R_{+}\right|+\left|R_{-}\right|} \frac{\left(z_{\text {fund }}\left(R_{+}\right)\right)^{2}\left(z_{\text {fund }}\left(R_{-}\right)\right)^{2}}{z_{\text {vect }}\left(R_{+}, R_{-}\right)} .
$$

However, this is not the Nekrasov function featuring in the AGT correspondence but a spectral dual [26-28] thereof. One can also notice that the expansion in eq. (1.7) is not in the original coupling constant $\Lambda$, which now enters each term in a nontrivial way, but in the dual coupling $\Lambda^{\vee}$, i.e. the momentum $q^{\alpha}$ of the conformal block.

We therefore have two different expansions of the $q$-deformed conformal block connected by the spectral duality. The original expansion in terms of a special basis corresponds to the AGT dual Nekrasov function and the spectral dual expansion in the intermediate momentum corresponds to the manifest sum over poles in the DF integrals:

$$
\begin{gathered}
\mathcal{B}\left(\Lambda \mid \begin{array}{ccc}
\alpha_{\Lambda} & & \alpha_{1} \\
\alpha_{0} & \alpha & \alpha_{\infty}
\end{array}\right)=Z_{\text {Spectral }}^{\mathrm{AGT}} \\
Z_{\mathrm{Nek}}^{\vee}\left(\Lambda^{\vee}=q^{2 \alpha} \mid a^{\vee}, m_{\left.m_{f}^{\vee}, q, t\right)}^{\mathrm{DF}}\right.
\end{gathered}
$$

Generally the spectral dual Nekrasov functions describe gauge theories with different gauge groups and matter content: $\mathrm{SU}(N)^{M-1}$ and $\mathrm{SU}(M)^{N-1}$ quiver theories respectively [29]. In the case at hand $N=M=2$ so the dual gauge groups are in fact the same. Nevertheless, the parameters of the theories are reshuffled by the duality, e.g. the dual coupling constant $\Lambda^{\vee}$ is a combination of mass parameters of the original theory. Spectral duality plays a prominent role in the Seiberg-Witten integrable systems [30-34] associated with the gauge theories. One can see that the AGT relation is a combination of the explicit DF expansion [24, 25] and the spectral duality.

We can fill the missing corner in the diagram (1.9) by taking the AGT dual of $Z_{\text {Nek }}^{\vee}$ or interpreting $Z_{\mathrm{Nek}}$ as a DF expansion of a dual conformal block $\mathcal{B}^{\vee}$ :

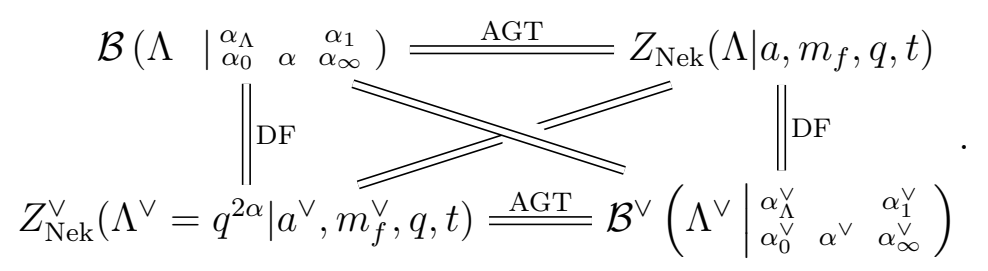



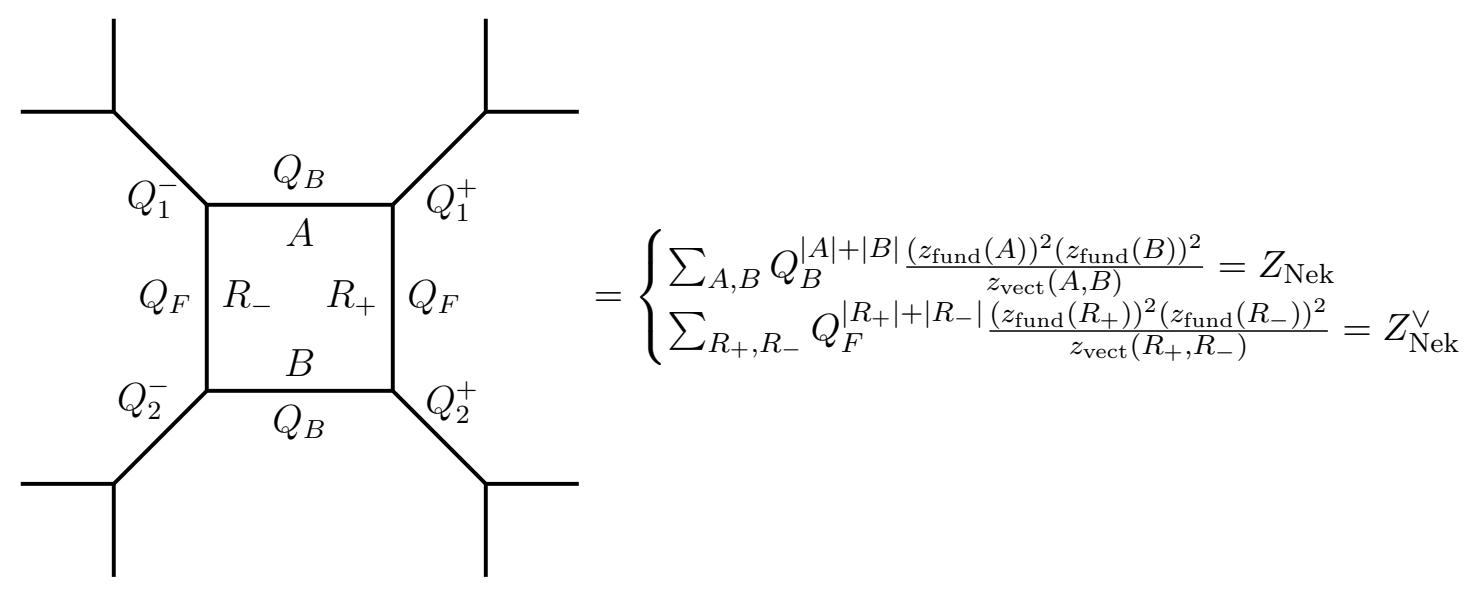

Figure 1. Toric diagram engineering an $\mathrm{SU}(2)$ gauge theory with four fundamental hypermultiplets. $Q_{F}$ corresponds to the vev of the scalar, $Q_{1,2}^{ \pm}$represent the masses of the hypermultiplets and $Q_{B} \sim \Lambda$ is the coupling constant of the theory.

Generally the spectral dual conformal block $\mathcal{B}^{\vee}$ has different number of points and different conformal algebra ( $q$ - $W_{M}$ instead of $q$-Virasoro) compared to the original one $\mathcal{B}$. Again, the case we consider here is extremely simple: the number of points and the algebra remain the same for the four-point conformal block of $q$-Virasoro. The dimensions of the fields and their coordinates are however expressed through each other in a nontrivial way. We will mention a tentative application of this duality for conformal blocks in section 7 .

Why do these new duality features become visible only in the five dimensional version of the AGT correspondence and not in the original one? It turns out the in five dimensions gauge theory partition function has fine structure, which can be effectively analysed from the topological string theory point of view. In the geometric engineering approach $[35,36]$, the $\mathcal{N}=1$ gauge theory in five dimensions is obtained by compactifying M-theory on a certain toric Calabi-Yau threefold. Five dimensional Nekrasov partition function is equal to the topological string partition function on the threefold which geometrically engineers the gauge theory. ${ }^{1}$ The geometry of this manifold is encoded in its toric diagram. For our case of $\mathrm{SU}(2)$ gauge theory with four fundamental hypermultiplets the diagram is shown in figure 1. The edges of the diagram correspond to the two-cycles in the threefold while Kähler parameters $Q_{i}$ of these cycles correspond to the gauge theory parameters.

The topological string partition function can be computed using the topological vertex technique $[39,40]$. To each edge of the diagram one assigns a partition and to each trivalent vertex one assigns a certain expression $C_{\lambda \mu \nu}(q)$ depending on three partitions residing on the adjacent edges. External edges carry empty partitions. Partition function is obtained by summing over all the partitions with weights $\left(-Q_{i}\right)^{\left|\lambda_{i}\right|}$.

There are essentially two ways to carry out the sum over partitions in figure 1 . One can cut the diagram vertically, compute the sum over $R_{ \pm}$explicitly and leave the sum over $A, B$ in the final answer. This sum over pairs of partitions is nothing but the sum in the

\footnotetext{
${ }^{1}$ The original geometric engineering gives the Nekrasov function in the self-dual $\Omega$-background, i.e. $t=q$. To get $t \neq q$ one should consider the refined topological strings [37, 38].
} 
Nekrasov function $Z_{\mathrm{Nek}}$. Moreover, each half of the diagram corresponds to the matrix element in the conformal block expansion (1.2):

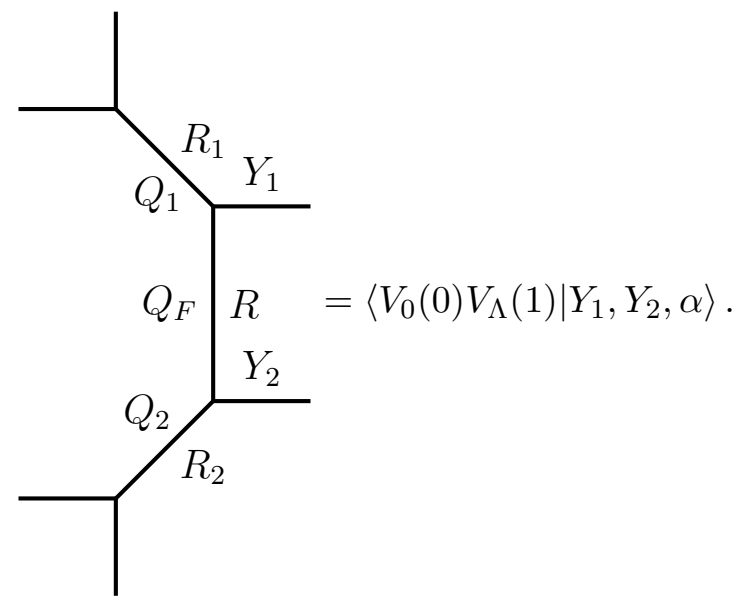

However there is one more way to perform the summation: one can cut the diagram horizontally and do the sums over $A$ and $B$ first. In this way one gets the spectral dual Nekrasov function $Z_{\mathrm{Nek}}^{\vee}$. The sum over $R_{ \pm}$corresponds to the sum in the DF integral representation (1.7):

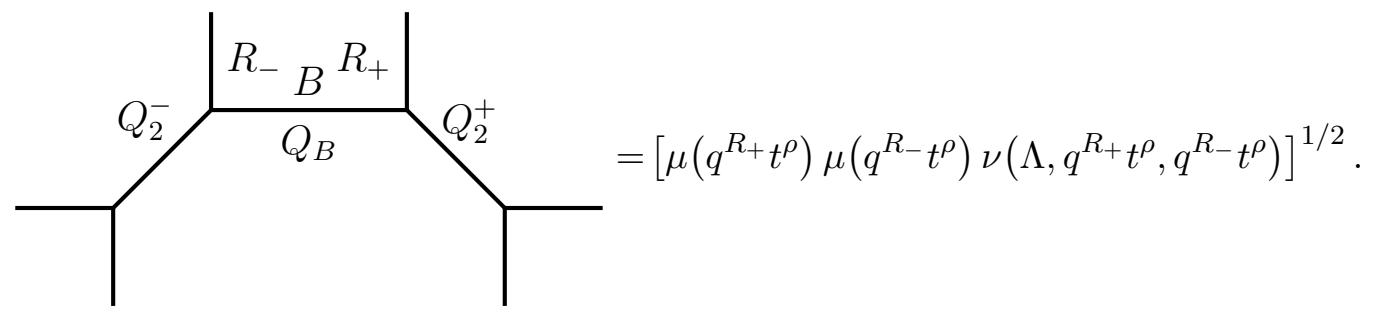

The spectral duality in this picture is natural and corresponds to taking the mirror image along the diagonal (or alternatively rotating the diagram by $\frac{\pi}{2}$ ).

In section 2 we introduce the generalized Macdonald Hamiltonian, compute its eigenfunctions, i.e. generalized Macdonald polynomials, and check their elementary properties. In section 3 we describe the $q$-deformation of the Dotsenko-Fateev integral representation for conformal blocks and introduce the decomposition in terms of generalized Macdonald polynomials. In section 4 we derive the loop equations for the corresponding $q$-deformed $\beta$-ensemble, find the averages of the generalized Macdonald polynomials and check the AGT correspondence. We prove the spectral duality for $q$-deformed Liouville correlators in section 5 and compare our results to the topological string partition function in section 6 . We present our conclusions in section 7 .

\section{Generalized Macdonald polynomials}

Generalized Macdonald polynomials are symmetric polynomials in two sets of variables $x_{i}$ and $\bar{x}_{i}$ labelled by pairs of partitions $Y_{1}, Y_{2}$ and depending on an extra parameter $Q$. Ordinary Macdonald polynomials can be obtained as the eigenfunctions of Macdonald 
Hamiltonians (see appendix A). Similarly, generalized Macdonald polynomials are eigenfunctions of the following generalized MacDonald operator, which can be thought of as the Ding-Iohara coproduct acting on the of the original Macdonald Hamiltonian [15]: ${ }^{2}$

$$
\begin{aligned}
H_{1}^{\text {gen }}=\frac{1}{t-1} \oint_{\mathcal{C}_{0}} \frac{d z}{z} & {\left[\left(e^{\sum_{n \geq 1} \frac{1-t^{-n}}{n} z^{n} p_{n}} e^{\sum_{n \geq 1}\left(q^{n}-1\right) z^{-n} \frac{\partial}{\partial p_{n}}}-1\right)+\right.} \\
+ & \left.Q\left(e^{\sum_{n \geq 1} \frac{1-t^{-n}}{n} z^{n}\left(\left(1-t^{n} q^{-n}\right) p_{n}+\bar{p}_{n}\right)} e^{\sum_{n \geq 1}\left(q^{n}-1\right) z^{-n} \frac{\partial}{\partial \bar{p}_{n}}}-1\right)\right] .
\end{aligned}
$$

The eigenvalues of the generalized Hamiltonian are given by the sums of ordinary Macdonald eigenvalues

$$
H_{1}^{\text {gen }} M_{Y^{1} Y^{2}}^{(q, t)}(Q \mid p, \bar{p})=\left\{\sum_{a=1}^{2} Q^{a-1} \sum_{i \geq 1}\left(q^{Y_{i}^{a}}-1\right) t^{-i}\right\} M_{Y^{1} Y^{2}}^{(q, t)}(Q \mid p, \bar{p}) .
$$

Eigenvalues of $H_{1}^{\text {gen }}$ are non-degenerate, so the eigenfunctions are uniquely determined. In practice one finds Macdonald polynomials by solving linear equations for the coefficients of the polynomial eigenfunctions. One writes a general symmetric polynomial as a linear combination of $p_{Y}=p_{Y_{1}} p_{Y_{2}} \cdots p_{Y_{n}}$, e.g. $M_{Y}=\sum_{Y^{\prime}} c_{Y Y^{\prime}} p_{Y^{\prime}}$. Acting with $H_{1}^{\text {gen }}$ involves shifts of $p_{n}$ :

$$
\exp \left(\sum_{n \geq 1}\left(q^{n}-1\right) z^{-n} \frac{\partial}{\partial p_{n}}\right) f\left(p_{k}\right)=f\left(p_{k}+\left(q^{k}-1\right) z^{-k}\right) .
$$

This gives a rational function so it is straightforward to obtain the residues.

The properties of generalized Macdonald functions are analogous to those of generalized Jack polynomials, though with minor variations.

1. Orthogonality, conjugation and normalization. There is a convenient way to normalize generalized Macdonald polynomials: $M_{Y^{1} Y^{2}}^{(q, t)}(Q \mid p, \bar{p})=m_{Y^{1}}(p) m_{Y^{2}}(\bar{p})+$ $\sum_{\vec{W} \neq \vec{Y}} c_{\vec{Y} \vec{W}} m_{W^{1}}(p) m_{W^{2}}(\bar{p})$. Conjugate Macdonald polynomials can be expressed through Macdonald polynomials themselves as follows

$$
M_{Y^{1} Y^{2}}^{*(q, t)}\left(Q \mid p_{n}, \bar{p}_{n}\right)=M_{Y^{2} Y^{1}}^{(q, t)}\left(Q^{-1} \mid \bar{p}_{n}, p_{n}-\left(1-t^{n} / q^{n}\right) \bar{p}_{n}\right) .
$$

Notice that the term proportional to $1-t^{n} / q^{n}$ vanishes both for $t=q$ and in the four dimensional limit $t=q^{\beta}, q \rightarrow 1$. The polynomials form an orthogonal system:

$$
\left\langle M_{\vec{A}}^{*}, M_{\vec{B}}\right\rangle=\delta_{\vec{A} \vec{B}} \frac{C_{A^{1}} C_{A^{2}}}{C_{A^{1}}^{\prime} C_{A^{2}}^{\prime}}
$$

with respect to Macdonald scalar product

$$
\left\langle f\left(p_{k}\right), g\left(p_{k}\right)\right\rangle=\left.f\left(n \frac{1-q^{n}}{1-t^{n}} \frac{\partial}{\partial p_{k}}\right) g\left(p_{k}\right)\right|_{p_{k}=0} .
$$

\footnotetext{
${ }^{2}$ We rescale $\bar{p}_{n}$ by $(q / t)^{n / 2}$ compared to $[15]$.
} 
Here

$$
C_{A}=\prod_{(i, j) \in A}\left(1-q^{A_{i}-j+1} t^{A_{j}^{\mathrm{T}}-i}\right), \quad C_{A}^{\prime}=\prod_{(i, j) \in A}\left(1-q^{A_{i}-j} t^{A_{j}^{\mathrm{T}}-i+1}\right) .
$$

Sometimes it is more convenient to use a different normalization

$$
\begin{aligned}
& \widetilde{M}_{\vec{Y}}(p)=G_{Y^{2} Y^{1}}\left(Q^{-1}\right) \prod_{a=1,2} \prod_{(i, j) \in Y^{a}}\left(1-q^{Y_{i}^{a}-j} t^{\left(Y^{a}\right)_{j}^{\mathrm{T}}-i+1}\right) \widetilde{M}_{Y}(p), \\
& \widetilde{M}_{\vec{Y}}^{*}(p)=G_{Y^{1} Y^{2}}(Q)\left(\frac{t}{q}\right)^{|\vec{Y}|} \prod_{a=1,2} \prod_{(i, j) \in Y^{a}}\left(1-q^{-Y_{i}^{a}+j} t^{-\left(Y^{a}\right)_{j}^{\mathrm{T}}+i-1}\right) \widetilde{M}_{Y}^{*}(p),
\end{aligned}
$$

where $G_{A B}$ is given in appendix B. This normalization is tailored so that the norms of the polynomials are given by the vector part of the Nekrasov function:

$$
\left\langle\widetilde{M}_{\vec{Y}}^{*}(Q), \widetilde{M}_{\vec{W}}(Q)\right\rangle=z_{\text {vect }}(\vec{Y}, Q) \delta_{\vec{Y}, \vec{W}} .
$$

Notice also that in this normalization generalized Macdonald polynomials depend polynomially on the parameters $Q, q$ and $t$.

2. The "inversion" relation.

$$
M_{\vec{Y}}^{(q, t)}\left(Q \mid-\frac{1-q^{k}}{1-t^{k}} p_{k}^{(a)}\right)=(-1)^{|\vec{Y}|} \frac{C_{Y^{1}} C_{Y^{2}}}{C_{Y^{1}}^{\prime} C_{Y^{2}}^{\prime}} M_{\vec{Y}^{T}}^{\left(t^{-1}, q^{-1}\right)}\left(Q \mid p_{k}^{(a)}\right) .
$$

3. Cauchy completeness identity. Generalized Macdonald polynomials form a complete basis in the space of symmetric functions in two sets of variables:

$$
\begin{aligned}
\sum_{Y} \frac{C_{Y^{1}}^{\prime} C_{Y^{2}}^{\prime}}{C_{Y^{1}} C_{Y^{2}}} M_{\vec{Y}}^{*}\left(Q \mid p_{k}^{(a)}\right) M_{\vec{Y}}\left(Q \mid q_{k}^{(a)}\right) & =\sum_{\vec{Y}} \frac{\widetilde{M}_{\vec{Y}}^{*}\left(Q \mid p_{k}^{(a)}\right) \widetilde{M}_{\vec{Y}}\left(Q \mid q_{k}^{(a)}\right)}{z_{\text {vect }}(\vec{Y}, Q)}= \\
& =\exp \left(\sum_{k \geq 1} \sum_{a=1}^{2} \frac{1-t^{k}}{1-q^{k}} \frac{p_{k}^{(a)} q_{k}^{(a)}}{k}\right) .
\end{aligned}
$$

4. Specialization identities. For $q \rightarrow 1, t=q^{\beta}$ generalized Macdonald polynomials become generalized Jack polynomials $M_{\vec{Y}}^{(q, t)}\left(q^{2 a} \mid p_{k}\right) \rightarrow J_{\vec{Y}}^{(\beta)}\left(a \mid p_{k}\right)$. For $t=q$ the Hamiltonian $H_{1}^{\text {gen }}$ turns into a sum of screening factors. Therefore, the eigenfunctions become products of Schur polynomials: $M_{Y^{1} Y^{2}}^{(q, q)}\left(Q \mid p_{k}\right)=\chi_{Y^{1}}\left(p_{k}\right) \chi_{Y^{2}}\left(\bar{p}_{k}\right)$. This is consistent with the results of [19, 20], where Schur polynomials were found to be the right basis for the case of $t=q$.

One has the following reduction to ordinary Macdonald polynomials: $M_{Y^{1} Y^{2}}^{(q, t)}\left(Q \mid 0, \bar{p}_{k}\right)$ $=M_{Y^{2}}^{(q, t)}\left(\bar{p}_{k}\right)$ and also $M_{Y^{1} \emptyset}^{(q, t)}\left(Q \mid p_{k}, \bar{p}_{k}\right)=M_{Y^{1}}^{(q, t)}\left(p_{k}\right)$. The value of generalized Macdonald polynomials on the Weyl vector can also be found and are nicely expressed through the ordinary Macdonald polynomials:

$$
\begin{aligned}
M_{Y^{1} Y^{2}}^{(q, t)}\left(Q \mid p_{k}=\frac{1-Q^{n}}{1-t^{n}}, \bar{p}_{k}=-\frac{1-\frac{t^{n}}{q^{n}}}{1-t^{n}}\right) & =M_{Y^{1}}^{(q, t)}\left(p_{k}=\frac{1-Q^{n}}{1-t^{n}}\right), \\
M_{Y^{1} Y^{2}}^{(q, t)}\left(Q \mid p_{k}=0, \bar{p}_{k}=-\frac{1-Q^{n}}{1-t^{n}}\right) & =M_{Y^{2}}^{(q, t)}\left(p_{k}=-\frac{1-Q^{n}}{1-t^{n}}\right) .
\end{aligned}
$$


The first few generalized polynomials are

$$
\begin{aligned}
M_{[1],[]}= & p_{1} \\
M_{[],[1]}= & \bar{p}_{1}-\frac{p_{1}(q-t)}{q(Q-1)} \\
M_{[2],[]}= & \frac{(q+1)(t-1) p_{1}^{2}}{2(q t-1)}+\frac{(q-1)(t+1) p_{2}}{2(q t-1)} \\
M_{[1,1],[]}= & \frac{p_{1}^{2}}{2}-\frac{p_{2}}{2} \\
M_{[1],[1]}= & -\frac{p_{1}^{2}(q-t)(q t Q+q Q-t Q-2 t+Q)}{2 q(q Q-1)(Q-t)}+ \\
& +\frac{(q-1)(t+1) Q p_{2}(q-t)}{2 q(q Q-1)(Q-t)}+\bar{p}_{1} p_{1}, \\
& -\frac{(q+1)(t-1) p_{1}^{2}(q-t)\left(q^{2}+q t Q-q t-t Q\right)}{2 q^{2}(Q-1)(q t-1)(q-Q)}- \\
M_{[],[2]}= & \frac{(q-1)(t+1) p_{2}(q-t)\left(q^{2}-q t Q+q t-t Q\right)}{2 q^{2}(Q-1)(q t-1)(q-Q)} \\
& +\frac{(q+1)(t-1) \bar{p}_{1} p_{1}(q-t)}{(q t-1)(q-Q)}+\frac{(q+1)(t-1) \bar{p}_{1}^{2}}{2(q t-1)}+\frac{(q-1)(t+1) \bar{p}_{2}}{2(q t-1)} \\
& \frac{p_{1}^{2}(q-t)\left(q-t^{2} Q+t Q-t\right)}{2 q^{2}(Q-1)(t Q-1)}- \\
& -\frac{p_{2}(q-t)\left(q-t^{2} Q-t Q+t 1\right)}{2 q^{2}(Q-1)(t Q-1)}-\frac{\bar{p}_{1} p_{1}(q-t)}{q(t Q-1)}+\frac{\bar{p}_{1}^{2}}{2}-\frac{\bar{p}_{2}}{2} . \\
M_{[],[1,1]} &
\end{aligned}
$$

\section{$3 q$-deformed Dotsenko-Fateev integrals}

In the Dotsenko-Fateev approach conformal blocks are expressed in terms of multiple contour integrals of the degenerate field insertion. What is specific to the $q$-deformed case is that these integrals can be taken by residues and reduce to multiple Jackson $q$ integrals (1.4). We use the $q$-integral formalism which turns out to be more convenient throughout this paper. The four-point conformal block is given by the following integral ${ }^{3}$

$$
\begin{aligned}
\mathcal{B}= & \int_{0}^{1} d_{q}^{N_{+}} z \int_{0}^{\Lambda^{-1} q / t} d_{q}^{N_{-}} z \prod_{i \neq j} \prod_{k=0}^{\beta-1}\left(1-q^{k} \frac{z_{i}}{z_{j}}\right) \prod_{i=1}^{N_{+}+N_{-}} z_{i}^{\alpha_{0}} \prod_{k=0}^{v_{+}-1}\left(1-q^{k} z_{i}\right) \prod_{k=0}^{v_{-}-1}\left(1-q^{k} \Lambda \frac{t}{q} z_{i}\right)= \\
= & C \int_{0}^{1} d_{q}^{N_{+}} x \int_{0}^{1} d_{q}^{N_{-}} y \Delta^{(q, t)}(x) \prod_{i=1}^{N_{+}} x_{i}^{u_{+}} \prod_{k=0}^{v_{+}-1}\left(1-q^{k} x_{i}\right) \Delta^{(q, t)}(y) \prod_{i=1}^{N_{-}} y_{i}^{u_{-}} \prod_{k=0}^{v_{-}-1}\left(1-q^{k} y_{i}\right) \times \\
& \times \prod_{i=1}^{N_{+}} \prod_{j=1}^{N_{-}} \prod_{k=0}^{\beta-1}\left(1-q^{k} \Lambda \frac{t}{q} \frac{x_{i}}{y_{j}}\right)\left(1-q^{k} \Lambda \frac{x_{i}}{y_{j}}\right) \prod_{j=1}^{N_{-}} \prod_{n=0}^{v_{+}-1}\left(1-\Lambda q^{-n} \frac{t}{q} \frac{1}{y_{j}}\right) \prod_{i=1}^{N_{+}} \prod_{l=0}^{v_{-}-1}\left(1-\Lambda q^{l} \frac{t}{q} x_{i}\right),
\end{aligned}
$$

\footnotetext{
${ }^{3}$ We assume $v_{ \pm}, \beta$ to be integer, though all our formulas have well-defined analytic continuation to non-integer values.
} 
where $\Delta^{(q, t)}(x)=\prod_{k=0}^{\beta-1} \prod_{i \neq j}\left(x_{i}-q^{k} x_{j}\right), u_{+}=\alpha_{0}+\beta\left(1-N_{+}-N_{-}\right)$and $u_{-}=\alpha_{0}+v_{+}+$ $\beta\left(1+N_{+}-N_{-}\right)$.

The completeness (2.11) of the generalized Macdonald polynomials can be employed in the last line of eq. (3.1) and gives the following expansion

$$
\begin{aligned}
& \prod_{i=1}^{N_{+}} \prod_{j=1}^{N_{-}} \prod_{k=0}^{\beta-1}\left(1-q^{k} \Lambda \frac{t}{q} \frac{x_{i}}{y_{j}}\right)\left(1-q^{k} \Lambda \frac{x_{i}}{y_{j}}\right) \prod_{j=1}^{N_{-}} \prod_{n=0}^{v_{+}-1}\left(1-\Lambda q^{-n} \frac{t}{q} \frac{1}{y_{j}}\right) \prod_{i=1}^{N_{+}} \prod_{l=0}^{v_{-}-1}\left(1-\Lambda q^{l} \frac{t}{q} x_{i}\right)= \\
& =\exp \left\{\sum_{n \geq 1} \frac{\Lambda^{n}}{n} \frac{1-t^{n}}{1-q^{n}}\left[\left(-p_{n} \frac{t^{n}}{q^{n}}-\frac{1-q^{-n v_{+}}}{1-t^{-n}}\right) q_{-n}+p_{n}\left(-q_{-n}-\left(\frac{t}{q}\right)^{n}\right) \frac{1-q^{n v_{-}}}{1-t^{n}}\right]\right\}= \\
& =\sum_{A, B} \Lambda^{|A|+|B|} \frac{C_{A} C_{B}}{C_{A}^{\prime} C_{B}^{\prime}} M_{A B}^{*(q, t)}\left(q^{2 a} \mid-\frac{t^{n}}{q^{n}} p_{n}-\frac{1-q^{-n v_{+}}}{1-t^{-n}}, p_{n}\right) \times \\
& \quad \times M_{A B}^{(q, t)}\left(q^{2 a} \mid q_{-n},-q_{-n}-\left(\frac{t}{q}\right)^{n} \frac{1-q^{n v_{-}}}{1-t^{n}}\right),
\end{aligned}
$$

where $p_{n}=\sum_{i=1}^{N_{+}} x_{i}^{n}, q_{n}=\sum_{j=1}^{N_{-}} y_{j}^{n}$ and $C_{A}, C_{A}^{\prime}$ are given by eq. (2.6). Observe that a new parameter $a$ has appeared in the last lines of eq. (3.2). This is an extra parameter of generalized Macdonald polynomials, which should be tuned to conform with the parameters of the Selberg average according to eqs. (B.3). To check the AGT correspondence (1.3) one should therefore check that

$$
\begin{aligned}
& \frac{C_{A^{1}} C_{A^{2}}}{C_{A^{1}}^{\prime} C_{A^{2}}^{\prime}}\left\langle M_{A^{1} A^{2}}^{*(q, t)}\right.\left.\left(q^{2 a} \mid-\frac{t^{n}}{q^{n}} p_{n}-\frac{1-q^{-n v_{+}}}{1-t^{-n}}, p_{n}\right)\right\rangle_{+} \times \\
& \times\left\langle M_{A^{1} A^{2}}^{(q, t)}\left(q^{2 a} \mid q_{-n},-q_{-n}-\frac{t^{n}}{q^{n}} \frac{1-q^{n v_{-}}}{1-t^{n}}\right)\right\rangle_{-} \stackrel{\mathrm{AGT}}{=} \\
& \stackrel{\mathrm{AGT}}{=} \frac{\prod_{i=1}^{2} \prod_{f=1}^{2} f_{A^{i}}^{+}\left(m_{f}^{+}+a_{i}\right) f_{A^{i}}^{-}\left(m_{f}^{-}+a_{i}\right)}{z_{\text {vect }}(A, a)}
\end{aligned}
$$

where

$$
\langle f(x)\rangle_{ \pm}=\frac{\int d_{q}^{N_{ \pm}} x \Delta^{(q, t)}(x) \prod_{i=1}^{N_{ \pm}} x_{i}^{u_{ \pm}} \prod_{k=0}^{v_{ \pm}-1}\left(q^{k} x_{i}-1\right) f(x)}{\int d_{q}^{N_{ \pm}} x \Delta^{(q, t)}(x) \prod_{i=1}^{N_{ \pm}} x_{i}^{u_{ \pm}} \prod_{k=0}^{v_{ \pm}-1}\left(q^{k} x_{i}-1\right)}
$$

and the parameters of the Selberg sum are identified with the gauge theory parameters with the help of eqs. (B.3). In the next section we develop the loop equations for the $q$-deformed beta-ensemble (3.4) in order to check eq. (3.3).

\section{Loop equations for $q$-deformed beta-ensemble}

Let us write down the loop equations for the DF integral. They provide the recurrence relations for the $q$-deformed beta-ensemble averages of $p_{Y}=\prod_{i} p_{Y_{i}}$ and therefore determine the average of any symmetric function.

One first observes that the $q$-integral of a total $q$-derivative is zero

$$
\int_{0}^{1} d_{q} z \frac{1}{z}\left(1-q^{z \partial_{z}}\right) g(z)=0
$$


as long as $g(1)=0$. The loop equation is obtained from the simple identity

$$
\int d_{q}^{N} x \sum_{i=1}^{N} \frac{1}{x_{i}}\left(q^{x_{i} \partial_{i}}-1\right) x_{i}\left[\frac{x_{i}-q}{z-x_{i}} \prod_{j \neq i} \frac{x_{i}-t x_{j}}{x_{i}-x_{j}} \prod_{k=1}^{N}\left(x_{k}^{u} \prod_{a=0}^{v-1}\left(q^{a} x_{k}-1\right)\right) \Delta^{q, t}(x) f(x)\right]=0
$$

where $f(x)$ denotes a symmetric polynomial in $x_{i}$ corresponding to the insertion of extra vertex operators. Eq. (4.2) in this case is valid as a power series in negative powers ${ }^{4}$ of $z$. The $q$-derivative of the $q$-Vandermonde is given by

$$
q^{x_{i} \partial_{x_{i}}} \Delta^{q, t}(x)=\left[\left(\frac{t}{q}\right)^{N-1} \prod_{j \neq i} \frac{\left(q x_{i}-x_{j}\right)\left(t x_{i}-x_{j}\right)}{\left(x_{i}-x_{j}\right)\left(x_{i}-\frac{t}{q} x_{j}\right)}\right] \Delta^{q, t}(x) .
$$

Using this expression one can rewrite eq. (4.2) as follows:

$$
\left\langle\sum_{i=1}^{N}\left[\frac{t^{N-1} q^{u+1}\left(q^{v} x_{i}-1\right)\left(q^{x_{i} \partial_{i}} f(x)\right)}{z-q x_{i}} \prod_{j \neq i} \frac{t x_{i}-x_{j}}{x_{i}-x_{j}}-\frac{\left(x_{i}-q\right) f(x)}{q\left(z-x_{i}\right)} \prod_{j \neq i} \frac{x_{i}-t x_{j}}{x_{i}-x_{j}}\right]\right\rangle=0,
$$

where $\langle\ldots\rangle$ denotes the $q$-Selberg average. Let us cast this equation into a more convenient form. We first observe that the sum in eq. (4.4) can be written as a contour integral

$$
\begin{aligned}
&\left\langle\oint _ { \mathcal { C } _ { x } } \frac { d \xi } { \xi } \left[\frac{t^{N-1} q^{u+1}\left(q^{v} \xi-1\right) e^{\sum_{n>0}\left(q^{n}-1\right) \xi^{n} \frac{\partial}{\partial p_{n}}} f\left(p_{n}\right)}{z-q \xi} \prod_{j=1}^{N} \frac{t \xi-x_{j}}{\xi-x_{j}}+\right.\right. \\
&\left.\left.+\frac{(\xi-q) f\left(p_{n}\right)}{q(z-\xi)} \prod_{j=1}^{N} \frac{\xi-t x_{j}}{\xi-x_{j}}\right]\right\rangle=0
\end{aligned}
$$

where the contour $\mathcal{C}_{x}$ encircles all the points $x_{i}$. Deforming the contour we pick up the residues at $0, z, z / q$ and $\infty$. One can show that the residue at infinity contains only positive powers of $z$ and will not affect the recurrence relations for $p_{n}$, so this term can be safely dropped. Other residues give

$$
\begin{array}{ll}
\xi=0: & t^{N}\left(\frac{q^{u+1}}{t}+1\right)\left\langle f\left(p_{n}\right)\right\rangle+ \\
\xi=z: & \left(\frac{z}{q}-1\right)\left\langle f\left(p_{n}\right) \exp \left[\sum_{n>0} \frac{1-t^{n}}{n} z^{-n} p_{n}\right]\right\rangle+ \\
\xi=z / q: & t^{2 N-1} q^{u+1}\left(q^{v-1} z-1\right)\left\langle f\left(p_{n}+\left(1-q^{-n}\right) z^{n}\right) \exp \left[\sum_{n>0} \frac{1-t^{-n}}{n} q^{n} z^{-n} p_{n}\right]\right\rangle=0 .
\end{array}
$$

Analogously, assuming that $f(x)=f\left(p_{-n}\right)$ is a symmetric polynomial in negative powers of $x_{i}$ one expands eq. (4.2) in positive powers of $z$ and obtains the recurrence

\footnotetext{
${ }^{4}$ Positive powers give rise to total derivatives with nonvanishing boundary values.
} 
relations for the negative power sums $p_{-Y}=\prod_{i} p_{-Y_{i}}$ :

$$
\begin{aligned}
& \left\langle-\frac{1}{q}\left(t^{2 N-1} q^{u+v+1}+1\right) f\left(p_{-n}\right)+t^{N}\left(\frac{1}{q}-\frac{1}{z}\right) f\left(p_{-n}\right) \exp \left(\sum_{n \geq 1} \frac{z^{n}}{n}\left(1-t^{-n}\right) p_{-n}\right)+\right. \\
& \left.\quad+t^{N-1} q^{u+1}\left(q^{v-1}-\frac{1}{z}\right) f\left(p_{-n}+\left(1-q^{n}\right) z^{-n}\right) \exp \left(\sum_{n \geq 1} \frac{1}{n} z^{n} q^{-n}\left(1-t^{n}\right) p_{-n}\right)\right\rangle=0 .
\end{aligned}
$$

Let us demonstrate how the recurrence relations work for the simplest example. Considering $f\left(p_{n}\right)=1$ and expanding eq. (4.6) to zeroth order in $z^{-1}$ one obtains the average of $p_{1}$ :

$$
\left\langle p_{1}\right\rangle=\frac{q\left(t^{N}-1\right)\left(t^{N-1} q^{u+1}-1\right)}{(t-1)\left(t^{2 N-2} q^{u+v+2}-1\right)} .
$$

This agrees (for $Y=[1]$ ) with the expression for the average of Macdonald polynomials $[19,20]:^{5}$

$$
\begin{aligned}
\left\langle M_{Y}\left(p_{n}\right)\right\rangle & =\prod_{(i, j) \in Y} \frac{q t^{i-1}\left(1-t^{N-i+1} q^{j-1}\right)\left(1-q^{u+j} t^{N-i}\right)}{\left(1-t^{Y_{j}^{\mathrm{T}}-i+1} q^{Y_{i}-j}\right)\left(1-q^{u+v+j+1} t^{2 N-i-1}\right)}, \\
\left\langle M_{Y}\left(p_{-n}\right)\right\rangle & =\prod_{(i, j) \in Y} \frac{t^{i-N}\left(1-t^{N-i+1} q^{j-1}\right)\left(1-q^{u+v-j+2} t^{N+i-2}\right)}{q\left(1-t^{Y_{j}^{\mathrm{T}}-i+1} q^{Y_{i}-j}\right)\left(1-q^{u-j+1} t^{i-1}\right)} .
\end{aligned}
$$

The following simple relation for the negative power sums can be immediately deduced

$$
\left\langle p_{-Y}\right\rangle_{u, v, N}=q^{|Y|(1-v)}\left\langle p_{Y}\right\rangle_{-u-v-2+2 \beta-2 \beta N, v, N}
$$

One can also check that the averages of generalized Macdonald polynomials are given by the factorized expressions

$$
\begin{aligned}
& \left\langle M_{Y^{1} Y^{2}}^{(q, t)}\left(q^{-u_{-}-1} t \mid q_{-k},-q_{-k}-\left(\frac{t}{q}\right)^{k} \frac{1-q^{k v_{-}}}{1-t^{k}}\right)\right\rangle_{-}=\frac{1}{G_{Y^{2} Y^{1}}\left(t^{-1} q^{u_{-}+1}\right)} \times \\
& \quad \times \prod_{(i, j) \in Y^{1}} \frac{q^{1-j+u_{-}+v_{-}} t^{2 i-2}\left(t^{N_{-}-i+1} q^{j-1}-1\right)\left(t^{-N_{-}+2-i} q^{j-u_{-}-v_{-}-2}-1\right)}{q^{\left(Y^{1}\right)_{i}-j} t^{\left(Y^{1}\right)_{j}^{\mathrm{T}}-i+1}-1} \times \\
& \quad \times \prod_{(i, j) \in Y^{2}} \frac{\left(-q^{v_{-}-1} t^{i}\right)\left(t^{N_{-}-i} q^{u_{-}+j}-1\right)\left(t^{-N_{-}-i+2} q^{j-v_{-}-1}-1\right)}{q^{\left(Y^{2}\right)_{i}-j} t^{\left(Y^{2}\right)_{j}^{\mathrm{T}}-i+1}-1} \stackrel{(\mathrm{B} .3)}{=} \\
& \stackrel{(\mathrm{B} .3)}{=}(-1)^{\left|Y^{1}\right|} q^{\sum_{(i, j) \in Y^{1}}[j-2+\beta]+\sum_{(i, j) \in Y^{2}}[2 j-3-2 a+(2-i) \beta]} \prod_{k=1}^{2} \prod_{(i, j) \in Y^{k}}\left(1-q^{\left(Y^{k}\right)_{i}-j} t^{\left(Y^{k}\right)_{j}^{\mathrm{T}}-i+1}\right)^{-1} \times \\
& \quad \times \frac{\prod_{f=1}^{2} \prod_{k=1}^{2} f_{Y^{k}}^{-}\left(m_{f}^{-}+a_{k}\right)}{G_{Y^{2} Y^{1}}\left(q^{-2 a}\right)}
\end{aligned}
$$

\footnotetext{
${ }^{5}$ The overall scale differs by $q^{|Y|(1-v)}$. We have checked this identity up to the fourth level.
} 
and analogously

$$
\begin{aligned}
& \left\langle M_{Y^{1} Y^{2}}^{*(q, t)}\left(q^{-u_{+}-v_{+}-1} t^{1-2 N_{+}} \mid-\frac{t^{k}}{q^{k}} p_{k}-\frac{1-q^{-k v_{+}}}{1-t^{-k}}, p_{k}\right)\right\rangle_{+}=\frac{1}{G_{Y^{1} Y^{2}}\left(t^{-2 N_{+}+1} q^{-u_{+}-v_{+}-1}\right)} \times \\
& \times \prod_{(i, j) \in Y^{1}} \frac{\left(-q^{-3-u_{+}-2 v_{+}+2 j} t^{-2 N_{+}-i+3}\right)\left(t^{N_{+}+i-1} q^{v_{+}-j+1}-1\right)\left(t^{N_{+}+i-2} q^{u_{+}+v_{+}-j+2}-1\right)}{q^{\left(Y^{1}\right)_{i}-j} t^{\left(Y^{1}\right)_{j}^{\mathrm{T}}-i+1}-1} \times \\
& \times \prod_{(i, j) \in Y^{2}} \frac{q^{-u_{+}-v_{+}-j} t^{-2 N_{+}+2 i}\left(t^{N_{+}-i} q^{u_{+}+j}-1\right)\left(t^{N_{+}-i+1} q^{j-1}-1\right)}{q^{\left(Y^{2}\right)_{i}-j} t^{\left(Y^{2}\right)_{j}^{\mathrm{T}}-i+1}-1} \stackrel{(\mathrm{B} .3)}{=} \\
& \stackrel{\text { (B.3) }}{=}(-1)^{\left|Y^{2}\right|} q^{\sum_{(i, j) \in Y^{1}} \beta i+\sum_{(i, j) \in Y^{2}}(2 \beta i-\beta+2 a-j+1)} \times \\
& \times \prod_{k=1}^{2} \prod_{(i, j) \in Y^{k}}\left(1-q^{\left(Y^{k}\right)_{i}-j} t^{\left(Y^{k}\right)_{j}^{\mathrm{T}}-i+1}\right)^{-1} \frac{\prod_{f=1}^{2} \prod_{k=1}^{2} f_{Y^{k}}^{+}\left(m_{f}^{+}+a_{k}\right)}{G_{Y^{1} Y^{2}}\left(q^{2 a}\right)} .
\end{aligned}
$$

Combining eqs. (4.12), (4.13) we obtain the desired result (3.3), though with additional factor corresponding to the renormalization of the coupling constant $\Lambda \rightarrow\left(\frac{q}{t}\right)^{2} \Lambda$ :

$$
\begin{aligned}
\frac{C_{A^{1}} C_{A^{2}}}{C_{A^{1}}^{\prime} C_{A^{2}}^{\prime}}\left\langleM _ { A ^ { 1 } A ^ { 2 } } ^ { * ( q , t ) } \left( q^{2 a} \mid\right.\right. & \left.\left.-\frac{t^{n}}{q^{n}} p_{n}-\frac{1-q^{-n v_{+}}}{1-t^{n}}, p_{n}\right)\right\rangle_{+} \times \\
\times & \left\langle M_{A^{1} A^{2}}^{(q, t)}\left(q^{2 a} \mid q_{-n},-q_{-n}-\frac{1-q^{-n v_{-}}}{1-t^{-n}}\right)\right\rangle_{-}= \\
& =\left(\frac{q}{t}\right)^{2\left(\left|A^{1}\right|+\left|A^{2}\right|\right)} \frac{\prod_{i=1}^{2} \prod_{f=1}^{2} f_{A^{i}}^{+}\left(m_{f}^{+}+a_{i}\right) f_{A^{i}}^{-}\left(m_{f}^{-}+a_{i}\right)}{z_{\text {vect }}(A, a)} .
\end{aligned}
$$

This relation gives the proof of the five dimensional AGT conjecture for four-point conformal blocks for general $q \neq t$. However, one should notice that it relies on the formulas (4.12), (4.13) for the $q$-Selberg averages. In appendix $\mathrm{C}$ we sketch some ideas which might lead to the proof of these identities. We have performed computerized checks of eqs. (4.12) and (4.13) on the first three levels.

\section{$5 \quad$ Spectral duality for conformal blocks}

In this section we demonstrate spectral duality for four-point $q$-deformed conformal blocks by employing the ideas of $[24,25]$. We write the DF integrals as explicit sums over partitions and use combinatorial identities to cast them into the form of Nekrasov function.

Let us examine the $q$-Selberg integral featuring in the DF representation of the $q$-deformed CFT (3.4)

$$
\int d_{q}^{N} x \Delta^{(q, t)}(x) f(x)=\left.\sum_{k \in \mathbb{N}^{N}} \Delta^{(q, t)}(x) f(x)\right|_{x_{i}=q^{k_{i}}}
$$

When the $q$-Vandermonde $\Delta^{(q, t)}$ is taken into account one realizes that $x_{i}$ should have the form $q^{R_{i}} t^{N-i}$ for some partition $R$. We therefore write the sum in the DF representation of the conformal block (3.1) as a sum over pairs of partitions

$$
\frac{\mathcal{B}}{B_{\emptyset \emptyset}}=\sum_{R_{+}, R_{-}} \frac{B_{R_{+} R_{-}}}{B_{\emptyset \emptyset}}
$$


where $B_{R_{+} R_{-}}$is the integrand from eq. (3.1) evaluated at $x_{i}=q^{R_{+, i}} t^{N_{+}-i}, y_{j}=q^{R_{-, j}} t^{N_{--j}}$. We normalize the sum so that the term corresponding to the empty diagram is the identity.

Using the identities from appendix D one immediately obtains a compact expression for $B_{R_{+} R_{-}} / B_{\emptyset \emptyset}$ (in the computation we multiply all $x_{i}$ and $y_{j}$ by a factor of $q$ which does not affect the final answer):

$$
\begin{aligned}
\frac{B_{R_{+} R_{-}}}{B_{\emptyset \emptyset}}=\prod_{(i, j) \in R_{+}} \frac{q^{u_{+}} t^{2 i-2}\left(1-q^{v_{+}+j} t^{N_{+}-i}\right)\left(1-q^{j-1} t^{N_{+}-i+1}\right)}{\left(q^{R_{+, i}-j} t^{R_{+, j}^{\mathrm{T}}-i+1}-1\right)\left(q^{R_{+, i}-j+1} t^{R_{+, j}^{\mathrm{T}}-i}-1\right)} \times \\
\quad \times\left(1-\Lambda q^{j-2} t^{N_{+}+2-i}\right)\left(1-\Lambda q^{j+v_{-}-1} t^{N_{+}-i+1}\right) \times \\
\times \prod_{(i, j) \in R_{-}} \frac{q^{u_{-}} t^{2 i-2}\left(1-q^{j+v_{-}} t^{N_{-}-i}\right)\left(1-q^{j-1} t^{N_{-}-i+1}\right)}{\left(q^{R_{-, i}-j} t^{R_{-, j}^{\mathrm{T}}-i+1}-1\right)\left(q^{R_{-, i}-j+1} t^{R_{-, j}^{\mathrm{T}}-i}-1\right)} \times \\
\quad \times\left(1-\Lambda q^{-j} t^{i-N_{-}}\right)\left(1-\Lambda q^{-j-v_{+}-1} t^{i-N_{-}+1}\right) \times \\
\quad \times \frac{1}{G_{R_{+} R_{-}}\left(\Lambda \frac{t}{q} t^{N_{+}-N_{-}}\right) G_{R_{+} R_{-}}\left(\Lambda t^{N_{+}-N_{-}}\right)}= \\
=\left(\Lambda^{\vee}\right)^{\left|R_{+}\right|+\left|R_{-}\right|} \frac{\prod_{k= \pm} \prod_{f=1}^{2} f_{R_{k}}^{+}\left(a_{k}^{\vee}+m_{f}^{\vee,+}\right) f_{R_{k}}^{-}\left(a_{k}^{\vee}+m_{f}^{\vee,-}\right)}{z_{\mathrm{vect}}\left(R_{+}, R_{-}, a_{k}^{\vee}\right)},
\end{aligned}
$$

where $f^{ \pm}, G_{A B}$ and $z_{\text {vect }}$ are given in appendix B,

$$
\begin{aligned}
a_{+}^{\vee} & =-a_{-}^{\vee}=a^{\vee} \\
\Lambda^{\vee} & =\Lambda^{-1} q^{u_{+}+v_{+}+1} t^{N_{+}+N_{-}-2}=\Lambda^{-1} q^{u_{-}+1} t^{N_{-}-N_{+}-2}=\Lambda^{-1} q^{m_{2}^{-}-m_{2}^{+}} t^{-1}, \\
2 a^{\vee} & =\tau-1+\beta\left(N_{+}-N_{-}+1\right), \\
m_{1}^{\vee,+} & =a^{\vee}+v_{-}+1+\beta\left(N_{-}-1\right), \\
m_{2}^{\vee,+} & =a^{\vee}+\beta N_{-} \\
m_{1}^{\vee,-} & =a^{\vee}-\tau+1+\beta N_{-}-\beta, \\
m_{2}^{\vee,-} & =a^{\vee}-\tau+v_{+}+2+\beta N_{-}-2 \beta,
\end{aligned}
$$

and $\tau=\frac{\ln \Lambda}{\ln q}$. The last line of eq. (5.3) is manifestly given by the spectral dual Nekrasov function.

Since we have proven both horizontal and vertical equalities in eq. (1.9), the diagonal line should also be true. We therefore obtain a (slightly indirect) proof of the spectral duality for the $\mathrm{SU}(2)$ Nekrasov partition functions. Eventually, this implies that all the dualities in the diagram (1.10) are valid.

\section{Comparison with topological strings}

Let us now briefly discuss the meaning of our computations in the topological string theory framework. In this section we limit ourselves to the $t=q$ case.

Firstly we notice that the vertical half of the diagram from figure 1 indeed corresponds to the average of the generalized Macdonald polynomial (which for $t=q$ is just a product 
of Schur functions):

$$
\begin{gathered}
Q_{F} R=\prod_{k=1}^{2} \prod_{(i, j) \in Y_{k}} \frac{q^{2 i+m_{2}^{-}+a_{k}-j}}{1-q^{\left(Y^{k}\right)_{i}-j+\left(Y^{2}\right)_{j}^{\mathrm{T}}-i+1}} \frac{\prod_{f=1}^{2} f_{Y_{k}}^{-}\left(m_{f}^{-}+a_{k}\right)}{G_{Y_{1} Y_{2}}\left(q^{2 a}\right)}= \\
=(-1)^{\left|Y_{1}\right|} q^{3 \sum_{(i, j) \in Y_{1}}(i-j)+2 \sum_{(i, j) \in Y_{2}}(i-j)} Q_{1}^{\left|Y_{1}\right|+\left|Y_{2}\right|}\left\langle M_{Y_{1} Y_{2}}^{*}\right\rangle_{-}
\end{gathered}
$$

where

$$
Q_{F}=q^{2 a}, \quad Q_{1}^{-}=q^{-m_{1}^{-}-a}, \quad Q_{2}^{-}=q^{m_{2}^{-}-a} .
$$

This equality tells us that the vertical half of the toric diagram can be identified with the vertex operator insertion at point $z=1$ in the conformal block as announced in eq. (1.11).

Of course, the computations are the same for the horizontal halves of the diagram, which correspond to the DF integrands (see eq. (1.12)):

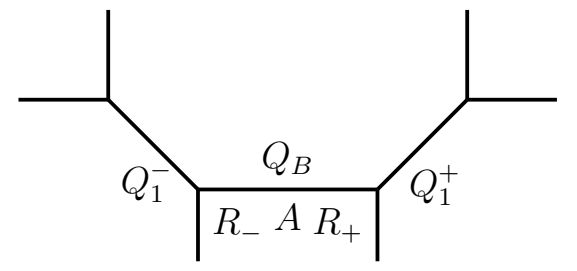

$$
=\frac{\prod_{k= \pm} \prod_{f=1}^{2} f_{R_{k}}^{+}\left(a_{k}^{\vee}+m_{f}^{\vee,+}\right) f_{R_{k}}^{+}\left(a_{k}^{\vee}+m_{f}^{\vee,-}\right)}{z_{\text {vect }}\left(R_{+}, R_{-}, a_{k}^{\vee}\right)}
$$

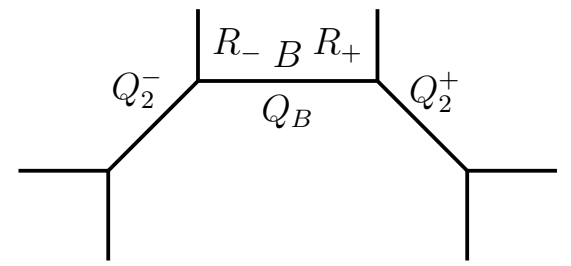

\section{Conclusions}

In this paper we have developed techniques to study $q$-deformed beta-ensemble and found the remarkable factorization formula for the averages of generalized Macdonald polynomials. We have proven the spectral duality for SU(2) Nekrasov functions and four-point conformal blocks by investigating the explicit DF integrals. We have also found the correspondence between the parts of the topological string partition function and the Liouville vertex operators.

It would be interesting to better understand other features of the CFT in the language of topological strings. In particular, the modular properties of conformal blocks should 
be encoded in the topological string partition function. The spectral duality in this case provides an alternative expansion, which can be used to find the nonperturbative results (cf. $[41,42]$ ).

Of course extensions of the above approach to the six dimensional gauge theories and to adjoint matter is desirable. Both of these generalizations can be obtained in the topological string by compactifying the toric diagram. The implications for the Seiberg-Witten elliptic integrable systems, most interestingly to the double elliptic ones [43, 44], should also be investigated.

\section{Acknowledgments}

The author would like to thank A. Mironov, Al. Morozov, And. Morozov, S. Shakirov and especially S. Mironov for stimulating discussions and criticism. The author appreciates the hospitality of the International Institute of Physics, Natal, Brazil where part of this work was done. The author is supported by the Russian Science Foundation grant 14-22-00161 and by D. Zimin's "Dynasty" foundation stipend.

\section{A Macdonald polynomials and Ruijsenaars Hamiltonians}

In this appendix we very briefly review some essential properties of the (trigonometric) Ruijsenaars Hamiltonians and list some useful expressions for them.

Macdonald polynomials are the eigenfunctions of the set of commuting Ruijsenaars Hamiltonians $H_{k}$, which can be thought of as a maximal commutative subalgebra inside the Ding-Iohara algebra [15]:

$$
H_{k} M_{Y}=e_{k}\left(\left\{\left(q^{Y_{i}}-1\right) t^{N-i}\right\}\right) M_{Y}
$$

where $e_{k}$ is the elementary symmetric polynomial of $N$ variables and

$$
\begin{aligned}
H_{k} & =\sum_{1 \leq i_{1}<\ldots<i_{k} \leq N} \frac{1}{\Delta(x)} t^{\sum_{a} x_{i_{a}} \partial_{i_{a}}} \Delta(x)\left(\frac{q}{t}\right)^{\sum_{a} x_{i_{a}} \partial_{i_{a}}}= \\
& =t^{\frac{k(k-1)}{2}} \sum_{1 \leq i_{1}<\ldots<i_{k} \leq N} \prod_{a=1}^{k} \prod_{j \neq i_{1} \ldots i_{k}} \frac{t x_{i_{a}}-x_{j}}{x_{i_{a}}-x_{j}} q^{\sum_{a} x_{i_{a}} \partial_{i_{a}}} .
\end{aligned}
$$

Note that for generic $q$ and $t$ the spectrum of $H_{1}$ is non-degenerate and no higher $H_{k}$ are needed to solve for the eigenfunctions. However, we give some expressions for higher $H_{k}$ for the sake of completeness.

Let us derive a compact expression for Ruijsenaars Hamiltonians acting on symmetric functions in terms of the power sum variables $p_{n}=\sum_{i=1}^{N} x_{i}^{n}$. The shift operator $q^{x_{i} \partial_{i}}$ in terms of power sums is given by

$$
q^{x_{i} \partial_{i}} f\left(p_{n}\right)=f\left(p_{n}+\left(q^{n}-1\right) x_{i}^{n}\right)=e^{\sum_{n \geq 1}\left(q^{n}-1\right) x_{i}^{n} \frac{\partial}{\partial p_{n}}} f\left(p_{n}\right) .
$$


The shift operators commute and we get

$$
H_{k}=t^{\frac{k(k-1)}{2}} \sum_{1 \leq i_{1}<\ldots<i_{k} \leq N} \prod_{a=1}^{k} \prod_{j \neq i_{1} \ldots i_{k}} \frac{t x_{i_{a}}-x_{j}}{x_{i_{a}}-x_{j}} \prod_{a=1}^{k} e^{\sum_{n \geq 1}\left(q^{n}-1\right) x_{i_{a}}^{n} \frac{\partial}{\partial p_{n}}} .
$$

One can see that this sum can be expressed as a sum over residues: ${ }^{6}$

$$
H_{k}=\frac{t^{\frac{k(k-1)}{2}}}{(t-1)^{k}[k]_{t} !} \oint_{\mathcal{C}_{x}} \prod_{a=1}^{k} \frac{d z_{a}}{z_{a}} \prod_{a<b} \frac{z_{a}-z_{b}}{z_{a}-t z_{b}} \prod_{i=1}^{N} \prod_{a=1}^{k} \frac{t z_{a}-x_{i}}{z_{a}-x_{i}} e^{\sum_{a=1}^{k} \sum_{n \geq 1}\left(q^{n}-1\right) z_{a}^{n} \frac{\partial}{\partial p_{n}}},
$$

where all $k$ integrals are taken over the same contour encircling the points $x_{i}$. The origin of the $t$-deformed factorial lies in the useful symmetrization formula

$$
\sum_{\sigma \in \mathfrak{S}_{k}} \prod_{i<j} \frac{t x_{\sigma(i)}-x_{\sigma(j)}}{x_{\sigma(i)}-x_{\sigma(j)}}=[k]_{t} ! .
$$

Using (A.6) once again for $z_{a}$ variables we get

$$
H_{k}=\frac{t^{\frac{k(k-1)}{2}}}{(t-1)^{k} k !} \oint_{\mathcal{C}_{x}} \prod_{a=1}^{k} \frac{d z_{a}}{z_{a}} \prod_{a \neq b} \frac{z_{a}-z_{b}}{z_{a}-t z_{b}} \prod_{i=1}^{N} \prod_{a=1}^{k} \frac{t z_{a}-x_{i}}{z_{a}-x_{i}} e^{\sum_{a=1}^{k} \sum_{n \geq 1}\left(q^{n}-1\right) z_{a}^{n} \frac{\partial}{\partial p_{n}}} .
$$

We expand the rational factors containing $x_{i}$ in terms of power sums

$H_{k}=\frac{t^{\frac{k(k-1)}{2}+k N}}{(t-1)^{k} k !} \oint_{\mathcal{C}_{x}} \prod_{a=1}^{k} \frac{d z_{a}}{z_{a}} \prod_{a \neq b} \frac{z_{a}-z_{b}}{z_{a}-t z_{b}} e^{\sum_{a=1}^{k} \sum_{n \geq 1} \frac{1-t^{-n}}{n} z_{a}^{-n} p_{n}} e^{\sum_{a=1}^{k} \sum_{n \geq 1}\left(q^{n}-1\right) z_{a}^{n} \frac{\partial}{\partial p_{n}}}$.

Let us now deform the integration contours so that they encircle $z=\infty$ and $z=0$. Additional residues arise when $z_{a}=t z_{b}$. For the first few integrals $H_{k}$ we have:

$$
\begin{aligned}
& H_{1}=h_{[1]}, \\
& H_{2}=\frac{1}{2} h_{[1,1]}-\frac{1}{2} h_{[2]}, \\
& H_{3}=\frac{1}{6} h_{[1,1,1]}-\frac{1}{2} h_{[2,1]}+\frac{1}{3} h_{[3]},
\end{aligned}
$$

where $^{7}$

$$
\begin{aligned}
h_{\left[1^{k}\right]}= & \frac{t^{k N+\frac{k(k-1)}{2}}}{(t-1)^{k}}\left(\oint_{\mathcal{C}_{\infty}}-\oint_{\mathcal{C}_{0}}\right)^{k} \prod_{a=1}^{k} \frac{d z_{a}}{z_{a}} \prod_{a<b} \frac{\left(z_{a}-z_{b}\right)^{2}}{\left(t z_{a}-z_{b}\right)\left(z_{a}-t z_{b}\right)} \times \\
& \times e^{\sum_{a=1}^{k}\left(\phi_{-}\left(z_{a}\right)-\phi_{-}\left(t z_{a}\right)\right)} e^{\sum_{a=1}^{k}\left(\phi_{+}\left(q z_{a}\right)-\phi_{+}\left(z_{a}\right)\right)} \\
h_{[k]}= & \frac{t^{k N}}{\left(t^{k}-1\right)}\left(\oint_{\mathcal{C}_{\infty}}-\oint_{\mathcal{C}_{0}}\right) \frac{d z}{z} e^{\phi_{-}(z)-\phi_{-}\left(t^{k} z\right)} e^{\sum_{a=0}^{k-1}\left(\phi_{+}\left(q t^{a} z\right)-\phi_{+}\left(t^{a} z\right)\right)} \\
h_{[2,1]}= & \frac{t^{3 N+1}}{(t-1)\left(t^{2}-1\right)}\left(\oint_{\mathcal{C}_{\infty}}-\oint_{\mathcal{C}_{0}}\right)^{2} \frac{d z_{1}}{z_{1}} \frac{d z_{2}}{z_{2}} \frac{\left(z_{1}-z_{2}\right)\left(t z_{1}-z_{2}\right)}{\left(z_{1}-t z_{2}\right)\left(t^{2} z_{1}-z_{2}\right)} \times \\
& \times e^{\phi_{-}\left(z_{1}\right)-\phi_{-}\left(t^{2} z_{1}\right)+\phi_{-}\left(z_{2}\right)-\phi_{-}\left(t z_{2}\right)} e^{\phi_{+}\left(q z_{1}\right)-\phi_{+}\left(z_{1}\right)+\phi_{+}\left(q t z_{1}\right)-\phi_{+}\left(t z_{1}\right)+\phi_{+}\left(q z_{2}\right)-\phi_{+}\left(z_{2}\right)}
\end{aligned}
$$

\footnotetext{
${ }^{6}$ We will always include the multiple of $\frac{1}{2 \pi i}$ into the definition of the contour integral.

${ }^{7}$ To be precise one should ensure the convergence of the expansions. We assume that $z_{a}$ are radially ordered, i.e. $\left|z_{1}\right|<\ldots<\left|z_{k}\right|$.
} 
where $\phi_{+}(z)=\sum_{n \geq 1} z^{n} \frac{\partial}{\partial p_{n}}$ and $\phi_{-}(z)=\sum_{n \geq 1} \frac{z^{-n}}{n} p_{n}$. Let us also note that $H_{k}$ can be understood as the commutative subalgebra in the Ding-Iohara vertex algebra.

$\boldsymbol{t}=\boldsymbol{q}$ case. $\boldsymbol{G L}(\infty)$ Casimir operators. In the limit $q=t$ Macdonald polynomials degenerate into Shur polynomials which are independent of $q$. In this limit all the Hamiltonians can be explicitly expressed through the first one. For example:

$$
\begin{aligned}
h_{\left[1^{k}\right]}(q) & =\left(h_{[1]}(q)\right)^{k}, \\
h_{[k]}(q) & =h_{[1]}\left(q^{k}\right), \\
h_{[2,1]}(q) & =h_{[1]}\left(q^{2}\right) h_{[1]}(q) .
\end{aligned}
$$

The first integral also simplifies and becomes

$$
h_{[1]}(q)=\oint_{\mathcal{C}_{\infty}} \frac{d z}{z}: e^{\phi(z)} \frac{q^{N+z \partial_{z}}-1}{q-1} e^{-\phi(z)}:
$$

where $\phi(z)=\phi_{-}(z)-\phi_{+}(z)$ and the normal ordering acts on $p_{n}$ and $\frac{\partial}{\partial p_{n}} \cdot 8$ Notice also that $h_{[1]}(q)$ with different values of $q$ commute:

$$
\left[h_{[1]}(q), h_{[1]}\left(q^{\prime}\right)\right]=0 .
$$

The values of $h_{[1]}(q)$ are expressed through the Casimir operators for the group $G L(\infty)$ :

$$
h_{[1]}(q) \chi_{R}=\left(q^{N-\frac{1}{2}} C_{R}(q)+\frac{q^{N}-1}{q-1}\right) \chi_{R},
$$

where $C_{R}\left(e^{\hbar}\right)=\sum_{i \geq 1}\left(e^{\hbar\left(R_{i}-i+\frac{1}{2}\right)}-e^{\hbar\left(-i+\frac{1}{2}\right)}\right)=\sum_{n \geq 0} \frac{\hbar^{n}}{n !} C_{R}(n)$ is the generating function of the Casimirs $C_{R}(n)=\sum_{i \geq 1}\left[\left(R_{i}-i+\frac{1}{2}\right)^{n}-\left(-i+\frac{1}{2}\right)^{n}\right]$.

\section{B Five dimensional Nekrasov functions and AGT relations}

The Nekrasov partition function for the $\mathrm{SU}(N)$ theory with $N_{f}=2 N$ fundamental hypermultiplets is given by

$$
Z_{\mathrm{Nek}}^{5 \mathrm{~d}}=\sum_{\vec{A}} \Lambda^{|\vec{A}|} \frac{\prod_{i=1}^{N} \prod_{f=1}^{N} f_{A_{i}}^{+}\left(m_{f}^{+}+a_{i}\right) f_{A_{i}}^{-}\left(m_{f}^{-}+a_{i}\right)}{z_{\text {vect }}(\vec{A}, \vec{a})},
$$

where $f_{A}^{ \pm}(x)=\prod_{(i, j) \in A}\left(1-q^{ \pm x} t^{ \pm(i-1)} q^{\mp(j-1)}\right), z_{\text {vect }}(\vec{A}, \vec{a})=\prod_{i, j=1}^{N} G_{A_{i} A_{j}}^{(q, t)}\left(a_{i}-a_{j}\right)$ and

$$
\begin{aligned}
G_{A B}^{(q, t)}(x) & =\prod_{(i, j) \in A}\left(1-q^{x} q^{A_{i}-j} t^{B_{j}^{\mathrm{T}}-i+1}\right) \prod_{(i, j) \in B}\left(1-q^{x} q^{-B_{i}+j-1} t^{-A_{j}^{\mathrm{T}}+i}\right)= \\
& =\prod_{(i, j) \in B}\left(1-q^{x} q^{A_{i}-j} t^{B_{j}^{\mathrm{T}}-i+1}\right) \prod_{(i, j) \in A}\left(1-q^{x} q^{-B_{i}+j-1} t^{-A_{j}^{\mathrm{T}}+i}\right) .
\end{aligned}
$$

\footnotetext{
${ }^{8}$ One can fermionize the bosonic operators so that $: e^{\phi(z)}: \sim \bar{\psi}(z),: e^{-\phi(z)}: \sim \psi(z)$. This leads to the fermionic construction of $G L(\infty)$ Casimirs.
} 
The AGT relations for $N=2$ are:

$$
\begin{aligned}
u_{+} & =m_{1}^{+}-m_{2}^{+}-1+\beta, & u_{-} & =-1+\beta-2 a, \\
v_{+} & =-m_{1}^{+}-m_{2}^{+}, & v_{-} & =-m_{1}^{-}-m_{2}^{-}, \\
\beta n_{+} & =-a+m_{2}^{+}, & \beta n_{-} & =a+m_{2}^{-},
\end{aligned}
$$

where $a_{1}=-a_{2}=a$. Masses $m_{a}$, vevs $a_{i}$, radius $R_{5}$ of the fifth dimension and $\epsilon_{1,2}$ all have dimensions of mass. In this paper we set the overall mass scale so that $\epsilon_{1}=-b^{2}, \epsilon_{2}=1$ and $q=e^{R}$. The $t$ parameter in Macdonald polynomials is related to $q$ by $t=q^{\beta}$ with $\beta=b^{2}$.

\section{Ruijsenaars Hamiltonians and loop equations}

Let us rewrite the loop equations (4.4) in terms of the Ruijsenaars Hamiltonian (A.2).

We first write down a useful identity:

$$
\sum_{i=1}^{N} \frac{1}{z-x_{i}} \prod_{j \neq i} \frac{t x_{i}-x_{j}}{x_{i}-x_{j}}=\frac{1}{(1-t) z}\left[1-\prod_{j=1}^{N} \frac{t z-x_{j}}{z-x_{j}}\right]
$$

which is proven by expanding the right hand side as a sum over poles in $z$.

Using eq. (C.1), one can rewrite eq. (4.4) as follows

$$
\begin{aligned}
\left\langle-\frac{t^{N-1} q^{u+1}}{z} H_{1} f(x)+t^{N-1} q^{u+1}\left(z q^{v-1}-1\right)\right. & {\left[H_{1}, \sum_{n \geq 1} \frac{q^{n} z^{-n-1}}{q^{n}-1} p_{n}\right] f(x)+} \\
& \left.+\frac{z-q t^{N}}{q z(1-t)} f(x)-\frac{z-q}{q z(1-t)} \prod_{j=1}^{N} \frac{z-t x_{j}}{z-x_{j}} f(x)\right\rangle=0,
\end{aligned}
$$

where $H_{1}$ is the first Ruijsenaars Hamiltonian. ${ }^{9}$ The expansion of eq. (C.2) in negative powers of $z$ gives an infinite number of $q$-Virasoro constraints which determine the average of any symmetric polynomials in $x_{i}$. In fact, only two of the infinite family of constrains are needed. Indeed, the Virasoro generators $L_{n}$ with positive $n$ can be obtained by commuting $L_{1}$ and $L_{2}$. The same holds for the $q$-deformed case. We may therefore consider only the equations obtained from the $z^{-1}$ and $z^{-2}$ terms:

$$
\begin{gathered}
\left\langle-t^{N-1} q^{u+v} H_{1} f(x)+\frac{t^{N-1} q^{u+v+1}}{q-1}\left[H_{1}, p_{1}\right] f(x)+\frac{1-t^{N}}{1-t} f(x)-q^{-1} p_{1} f(x)\right\rangle=0 \\
\left\langle\frac{t^{N-1} q^{u+2}}{(q-1)^{2}}\left[H_{1}, q^{v} p_{2}-q(q-1) p_{1}\right] f(x)-\frac{1}{2 q}\left((1+t) p_{2}+(1-t) p_{1}^{2}+2 q p_{1}\right) f(x)\right\rangle=0 .
\end{gathered}
$$

\footnotetext{
${ }^{9}$ Curiously, quantum dilogarithm $\phi_{q}(x)$ appears in this formula: indeed $\sum_{n \geq 1} \frac{q^{n} z^{-n-1}}{q^{n}-1} p_{n}=$ $-\partial_{z} \sum_{i=1}^{N} \ln \phi_{q}\left(\frac{q x_{i}}{z}\right)$.
} 
For $f(x)$ a Macdonald polynomial the Hamiltonian $H_{1}$ acts diagonally. However multiplication with $p_{n}$ produces a sum over multiple Macdonald polynomials. To see this one should use the Pieri idenity:

$$
e_{k}(p) M_{Y}(p)=\chi_{\left[1^{k}\right]}(p) M_{Y}(p)=M_{\left[1^{k}\right]}(p) M_{Y}(p)=\sum_{W} \prod_{(i, j) \in\left(C_{W \backslash Y} \backslash \backslash\left(R_{W \backslash Y}\right)\right.} \frac{b_{W}(i, j)}{b_{Y}(i, j)} M_{W}(p)
$$

where the sum is over diagrams $W$ such that the skew diagram $W \backslash Y$ is a horizontal strip (i.e. there are no more then one box belonging to each column) of $k$ boxes; $C_{W \backslash Y}$ (resp. $R_{W \backslash Y}$ ) is the set of columns (resp. rows) intersecting $W \backslash Y$ and

$$
b_{Y}(i, j)= \begin{cases}\frac{1-q^{Y_{i}-j} t^{Y_{j}^{\mathrm{T}}-i+1}}{1-q^{Y_{i}-j+1} t^{Y_{j}^{\mathrm{T}}-i}} & \text { if }(i, j) \in Y \\ 1 & \text { else. }\end{cases}
$$

Using Pieri formula for $k=1,2$ one can turn eqs. (C.3), (C.4) into a recurrence relations completely in terms of the averages of Macdonald polynomials. We are sure that one can obtain a proof of the identities (4.9), (4.10), (4.12), (4.13) along these lines, though we were not able to find it.

\section{Useful identities}

In this appendix we list some useful combinatorial identities related to Nekrasov functions.

$$
\begin{aligned}
\prod_{i=1}^{N} \prod_{k=0}^{v-1} \frac{1-Q q^{k+R_{i}} t^{-i}}{1-Q q^{k} t^{-i}} & =\prod_{(i, j) \in R} \frac{1-Q q^{v+j-1} t^{-i}}{1-Q q^{j-1} t^{-i}} \\
\frac{\Delta^{(q, t)}\left(q^{R_{i}} t^{-i}\right)}{\Delta^{(q, t)}\left(t^{-i}\right)} & =\prod_{k=0}^{\beta-1} \prod_{i=1}^{N} \prod_{j \neq i} \frac{q^{k+R_{i}} t^{-i}-q^{R_{j}} t^{-j}}{q^{k} t^{-i}-t^{-j}}= \\
& =\prod_{(i, j) \in R} \frac{t^{2(i-1)}\left(1-t^{N-i} q^{j}\right)\left(1-t^{N-i+1} q^{j-1}\right)}{\left(1-q^{R_{i}-j} t^{R_{j}^{\mathrm{T}}-i+1}\right)\left(1-q^{R_{i}-j+1} t^{R_{j}^{\mathrm{T}}-i}\right)}= \\
& =M_{R}^{(q, t)}\left(\frac{1-t^{n N}}{1-t^{n}}\right) M_{R^{\mathrm{T}}}^{(t, q)}\left(\frac{1-t^{n(1-N)} q^{-n}}{1-q^{n}}\right) \\
\prod_{k=0}^{\beta-1} \prod_{i=1}^{N_{+}} \prod_{j=1}^{N_{-}} \frac{1-Q q^{k+R_{i}-P_{j}} t^{j-i}}{1-Q q^{k} t^{j-i}} & =\frac{\prod_{(i, j) \in R}\left(1-Q q^{j-1} t^{N_{-}+1-i}\right) \prod_{(i, j) \in P}\left(1-Q q^{-j} t^{-N_{+}+i}\right)}{G_{R P}^{(q, t)}(Q)} \\
\prod_{(i, j) \in R}\left(1-q^{R_{i}-j+1} t^{R_{j}^{\mathrm{T}}-i}\right) & \left(1-q^{R_{i}-j} t^{R_{j}^{\mathrm{T}}-i+1}\right)=G_{R R}(1) \prod_{(i, j) \in R}\left(-q^{j} t^{i-1}\right) \\
G_{A B}(Q) & =G_{B A}\left(\frac{q}{t Q}\right) \prod_{(i, j) \in A}\left(-Q q^{j-1} t^{1-i}\right) \prod_{(i, j) \in B}\left(-Q q^{-j} t^{i}\right)
\end{aligned}
$$

where $G_{R P}$ is given by eq. (B.2). 
Open Access. This article is distributed under the terms of the Creative Commons Attribution License (CC-BY 4.0), which permits any use, distribution and reproduction in any medium, provided the original author(s) and source are credited.

\section{References}

[1] L.F. Alday, D. Gaiotto and Y. Tachikawa, Liouville Correlation Functions from Four-dimensional Gauge Theories, Lett. Math. Phys. 91 (2010) 167 [arXiv:0906.3219] [INSPIRE].

[2] D. Gaiotto, $N=2$ dualities, JHEP 08 (2012) 034 [arXiv:0904.2715] [INSPIRE].

[3] N. Wyllard, $A(N-1)$ conformal Toda field theory correlation functions from conformal $N=2 \mathrm{SU}(N)$ quiver gauge theories, JHEP 11 (2009) 002 [arXiv:0907.2189] [INSPIRE].

[4] A. Mironov and A. Morozov, On AGT relation in the case of U(3), Nucl. Phys. B 825 (2010) 1 [arXiv: 0908. 2569] [INSPIRE].

[5] R. Dijkgraaf and C. Vafa, Toda Theories, Matrix Models, Topological Strings and $N=2$ Gauge Systems, arXiv:0909.2453 [INSPIRE].

[6] H. Itoyama, K. Maruyoshi and T. Oota, The Quiver Matrix Model and 2d-4d Conformal Connection, Prog. Theor. Phys. 123 (2010) 957 [arXiv:0911.4244] [INSPIRE].

[7] T. Eguchi and K. Maruyoshi, Seiberg-Witten theory, matrix model and AGT relation, JHEP 07 (2010) 081 [arXiv: 1006.0828] [inSPIRE].

[8] T. Eguchi and K. Maruyoshi, Penner Type Matrix Model and Seiberg-Witten Theory, JHEP 02 (2010) 022 [arXiv:0911.4797] [INSPIRE].

[9] R. Schiappa and N. Wyllard, An Ar threesome: Matrix models, $2 d$ CFTs and $4 d N=2$ gauge theories, J. Math. Phys. 51 (2010) 082304 [arXiv:0911.5337] [INSPIRE].

[10] A. Mironov, A. Morozov and S. Shakirov, Matrix Model Conjecture for Exact BS Periods and Nekrasov Functions, JHEP 02 (2010) 030 [arXiv:0911.5721] [INSPIRE].

[11] A. Mironov, A. Morozov and S. Shakirov, Conformal blocks as Dotsenko-Fateev Integral Discriminants, Int. J. Mod. Phys. A 25 (2010) 3173 [arXiv:1001.0563] [InSPIRE].

[12] A. Mironov, A. Morozov and A. Morozov, Conformal blocks and generalized Selberg integrals, Nucl. Phys. B 843 (2011) 534 [arXiv:1003.5752] [INSPIRE].

[13] H. Awata and Y. Yamada, Five-dimensional AGT Conjecture and the Deformed Virasoro Algebra, JHEP 01 (2010) 125 [arXiv:0910.4431] [INSPIRE].

[14] H. Awata and Y. Yamada, Five-dimensional AGT Relation and the Deformed beta-ensemble, Prog. Theor. Phys. 124 (2010) 227 [arXiv:1004.5122] [INSPIRE].

[15] H. Awata, B. Feigin, A. Hoshino, M. Kanai, J. Shiraishi and S. Yanagida, Notes on Ding-Iohara algebra and AGT conjecture, arXiv:1106.4088 [INSPIRE].

[16] V.A. Alba, V.A. Fateev, A.V. Litvinov and G.M. Tarnopolskiy, On combinatorial expansion of the conformal blocks arising from AGT conjecture, Lett. Math. Phys. 98 (2011) 33 [arXiv: 1012.1312] [INSPIRE].

[17] V.A. Fateev and A.V. Litvinov, Integrable structure, W-symmetry and AGT relation, JHEP 01 (2012) 051 [arXiv:1109.4042] [INSPIRE]. 
[18] A. Belavin and V. Belavin, AGT conjecture and Integrable structure of Conformal field theory for $c=1$, Nucl. Phys. B 850 (2011) 199 [arXiv: 1102.0343] [INSPIRE].

[19] A. Mironov, A. Morozov and S. Shakirov, A direct proof of AGT conjecture at $\beta=1$, JHEP 02 (2011) 067 [arXiv: 1012.3137] [INSPIRE].

[20] A. Mironov, A. Morozov, S. Shakirov and A. Smirnov, Proving AGT conjecture as HS duality: extension to five dimensions, Nucl. Phys. B 855 (2012) 128 [arXiv:1105.0948] [INSPIRE].

[21] A. Morozov and A. Smirnov, Towards the Proof of AGT Relations with the Help of the Generalized Jack Polynomials, Lett. Math. Phys. 104 (2014) 585 [arXiv:1307.2576] [INSPIRE].

[22] S. Mironov, A. Morozov and Y. Zenkevich, Generalized Jack polynomials and the AGT relations for the $\mathrm{SU}(3)$ group, JETP Lett. 99 (2014) 109 [arXiv:1312.5732] [INSPIRE].

[23] Y. Ohkubo, Existence and Orthogonality of Generalized Jack Polynomials and Its q-Deformation, arXiv: 1404.5401 [INSPIRE].

[24] M. Aganagic, N. Haouzi, C. Kozcaz and S. Shakirov, Gauge/Liouville Triality, arXiv:1309.1687 [INSPIRE].

[25] M. Aganagic, N. Haouzi and S. Shakirov, $A_{n}$-Triality, arXiv:1403.3657 [INSPIRE].

[26] A. Mironov, A. Morozov, Y. Zenkevich and A. Zotov, Spectral Duality in Integrable Systems from AGT Conjecture, JETP Lett. 97 (2013) 45 [arXiv:1204.0913] [INSPIRE].

[27] A. Mironov, A. Morozov, B. Runov, Y. Zenkevich and A. Zotov, Spectral Duality Between Heisenberg Chain and Gaudin Model, Lett. Math. Phys. 103 (2013) 299 [arXiv:1206.6349] [INSPIRE].

[28] A. Mironov, A. Morozov, B. Runov, Y. Zenkevich and A. Zotov, Spectral dualities in XXZ spin chains and five dimensional gauge theories, JHEP 12 (2013) 034 [arXiv:1307.1502] [INSPIRE].

[29] L. Bao, E. Pomoni, M. Taki and F. Yagi, M5-Branes, Toric Diagrams and Gauge Theory Duality, JHEP 04 (2012) 105 [arXiv: 1112.5228] [INSPIRE].

[30] A. Gorsky, I. Krichever, A. Marshakov, A. Mironov and A. Morozov, Integrability and Seiberg-Witten exact solution, Phys. Lett. B 355 (1995) 466 [hep-th/9505035] [INSPIRE].

[31] R. Donagi and E. Witten, Supersymmetric Yang-Mills theory and integrable systems, Nucl. Phys. B 460 (1996) 299 [hep-th/9510101] [INSPIRE].

[32] N.A. Nekrasov and S.L. Shatashvili, Quantization of Integrable Systems and Four Dimensional Gauge Theories, arXiv:0908.4052 [INSPIRE].

[33] A. Mironov and A. Morozov, Nekrasov Functions and Exact Bohr-Zommerfeld Integrals, JHEP 04 (2010) 040 [arXiv:0910.5670] [INSPIRE].

[34] A. Mironov and A. Morozov, Nekrasov Functions from Exact BS Periods: The Case of $\mathrm{SU}(N)$, J. Phys. A 43 (2010) 195401 [arXiv:0911.2396] [inSPIRE].

[35] S.H. Katz, A. Klemm and C. Vafa, Geometric engineering of quantum field theories, Nucl. Phys. B 497 (1997) 173 [hep-th/9609239] [INSPIRE].

[36] S. Katz, P. Mayr and C. Vafa, Mirror symmetry and exact solution of 4-D $N=2$ gauge theories: 1, Adv. Theor. Math. Phys. 1 (1998) 53 [hep-th/9706110] [INSPIRE]. 
[37] A. Iqbal, C. Kozcaz and C. Vafa, The refined topological vertex, JHEP 10 (2009) 069 [hep-th/0701156] [INSPIRE].

[38] M. Taki, Refined Topological Vertex and Instanton Counting, JHEP 03 (2008) 048 [arXiv:0710.1776] [INSPIRE].

[39] A. Iqbal, All genus topological string amplitudes and five-brane webs as Feynman diagrams, hep-th/0207114 [INSPIRE].

[40] M. Aganagic, A. Klemm, M. Mariño and C. Vafa, The topological vertex, Commun. Math. Phys. 254 (2005) 425 [hep-th/0305132] [INSPIRE].

[41] D. Galakhov, A. Mironov and A. Morozov, S-duality and Modular Transformation as a non-perturbative deformation of the ordinary pq-duality, JHEP 06 (2014) 050 [arXiv: 1311.7069] [INSPIRE].

[42] N. Nemkov, S-duality as Fourier transform for arbitrary $\epsilon_{1}, \epsilon_{2}$, arXiv:1307.0773 [INSPIRE].

[43] G. Aminov, A. Mironov, A. Morozov and A. Zotov, Three-particle Integrable Systems with Elliptic Dependence on Momenta and Theta Function Identities, Phys. Lett. B 726 (2013) 802 [arXiv: 1307.1465] [INSPIRE].

[44] G. Aminov, H.W. Braden, A. Mironov, A. Morozov and A. Zotov, Seiberg-Witten curves and double-elliptic integrable systems, JHEP 01 (2015) 033 [arXiv:1410.0698] [INSPIRE]. 